Pacific Journal of Mathematics

FIXED POINT SETS OF PLANO CONTINUA 


\title{
FIXED POINT SETS OF PEANO CONTINUA
}

\author{
JOHN R. MARTIN
}

For each positive integer $n=1,2, \cdots$, it is shown that there is an $(n+1)$-dimensional acyclic $L C^{n-1}$ continuum $X_{n}$ containing an $n$-dimensional sphere which is not the fixed point set of any self-map of $X_{n}$.

1. Introduction. A subset $A$ of a topological space $X$ is called a fixed point set of $X$ if there is a (continuous) map $f: X \rightarrow X$ such that $f(x)=x$ iff $x \in A$. If $X$ is Hausdorff, then $A$ is closed, and, clearly, every retract of $X$ is a fixed point set of $X$. It is possible that a space $X$ may have the property that each of its nonempty closed subsets is a fixed point set of $X$. The problem of determining which spaces have this property, called the complete invariance property by L. E. Ward, Jr. in [5], has been investigated by $\mathrm{H}$. Robbins, Helga Schirmer, and L. E. Ward, Jr. Some spaces known to have the complete invariance property include $n$-cells [1], dendrites [2], convex subsets of Banach spaces [5], compact manifolds without boundary [3], and all compact triangulable manifolds with or without boundary [4].

The general question as to what properties a space must satisfy to insure that it has the complete invariance property has not been resolved. In fact, in [5, p. 553] L. E. Ward, Jr. asks the following question.

Does every Peano continuum have the complete invariance property?

The purpose of this note is to show that even acyclic Peano continua which possess higher order local connectedness need not have the complete invariance property. Indeed, for each positive integer $n=1,2, \cdots$, we give an example of an $(n+1)$-dimensional acyclic $L C^{n-1}$ continuum $X_{n}$ which fails to have the complete invariance property. Moreover, $X_{n}$ contains an $n$-dimensional sphere which is not a fixed point set of $X_{n}$.

2. Notation and the construction of $X_{n}$. We shall let $E^{n}$ denote Euclidean $n$-dimensional space, and we shall consider $E^{m}$ to be canonically imbedded in $E^{n}$ if $m<n$. The closed unit ball in $E^{n+1}$ shall be denoted by $B^{n+1}$ and the boundary $\mathrm{Bd} B^{n+1}$ of $B^{n+1}$ shall be denoted by $S^{n}$.

Consider the rectangle in $E^{2} \subset E^{n+2}$ with vertices $(1,-1,0, \cdots 0)$, $(0,-1,0, \cdots, 0),(0,1,0, \cdots, 0),(1,1,0, \cdots, 0)$. Let $D$ denote the closed disk consisting of this rectangle together with its interior in 
$E^{2}$, and let $A$ denote the segment in $\mathrm{Bd} D$ with endpoints $(0,-1,0, \cdots, 0)$ and $(0,1,0, \cdots, 0)$. We shall let $C$ denote the closure of the curve in $E^{2}$ whose equation is given by $y=\sin \pi / x$ for $0<$ $x \leqq 1$.

Let $\left\{B_{t}^{n+1} \mid 1 \leqq t<\infty\right\}$ be a disjoint collection of $(n+1)$-dimensional balls in $E^{n+2}$ satisfying the following properties.

(1) $\bigcup_{1 \leqq t<\infty} B_{t}^{n+1}$ is homeomorphic to $B^{n+1} \times[1, \infty)$ under a homeomorphism which sends $B_{t}^{n+1}$ onto $B^{n+1} \times\{t\}$ for each $t$ in the half-open interval $[1, \infty)$.

(2) $\lim _{t \rightarrow \infty} \delta\left(B_{t}^{n+1}\right)=0$, where $\delta\left(B_{t}^{n+1}\right)$ denotes the diameter of $B_{t}^{n+1}$.

(3) For each $t$ in $[1, \infty)$,

$$
B_{t}^{n+1} \cap E^{2}=\left\{\left(\frac{1}{t}, \sin \pi t, 0, \cdots, 0\right)\right\} .
$$

Let $S_{t}^{n}=\mathrm{Bd} B_{t}^{n+1}$. If $J$ is an interval in the real line, we define

$$
B_{J}^{n+1}=\bigcup_{t \in J} B_{t}^{n+1} \text { and } S_{J}^{n}=\bigcup_{t \in J} S_{t}^{n} .
$$

Definition. For $n=1,2, \cdots$, we define

$$
X_{n}=D \cup S_{[1, \infty)}^{n} \text {. }
$$

Essentially, $S_{[1, \infty)}^{n}$ is obtained by taking a cone over an $n$-sphere, removing the vertex, and then winding the resulting tube of $n$ spheres in a " $\sin 1 / x$ " fashion in $E^{n+2}$ so as to converge to the limit interval $A$. This procedure is carried out so that the intersection of $E^{2}$ with the closure of $S_{[1, \infty)}^{n}$ is precisely $C$. The disk $D$ is then attached to the closure of $S_{[1, \infty)}^{n}$ along $C$ to obtain $X_{n}$.

3. The properties of $X_{n}$.

Property 1. Each $X_{n}$ is an $(n+1)$-dimensional acyclic continuum.

Proof. By acyclic we mean that $X_{n}$ has the Čech cohomology of a point. Since $X_{n}$ is clearly an $(n+1)$-dimensional continuum, we need only show that it is acyclic. But

$$
X_{n}=\bigcap_{t \geqq 1}\left(D \cup S_{[1, \infty)}^{n} \cup B_{[t, \infty)}^{n+1}\right)
$$

and, for each $t, D \cup B_{[t, \infty)}^{n+1}$ is a deformation retract of $D \cup S_{[1, \infty)}^{n} \cup$ $B_{[t, \infty)}^{n+1}$. Since $D \cup B_{[t, \infty)}^{n+1}$ is contractible, each of the spaces $D \cup S_{[1, \infty)}^{n} \cup$ $B_{[t, \infty)}^{n+\infty}$ is contractible. Therefore, by the continuity axiom for Cech cohomology, $X_{n}$ is acyclic. 
Property 2. Each $X_{n}$ is $L C^{n-1}$ at points of $A$, and locally contractible elsewhere.

Proof. Clearly, every point $p$ in $X_{n}-A$ has a neighborhood which is finitely triangulable, and therefore $X_{n}$ is locally contractible at $p$. If $p \in A$, then $p$ has a compact neighborhood consisting of a closed disk $K$ and infinitely many disjoint cylinders of the form $S_{[t, t+\lambda]}^{n}$, each of which intersects $K$ in an arc. It then follows that $X_{n}$ is $L C^{n-1}$ at $p$.

Property 3. $S_{1}^{n}$ is not a fixed point set of $X_{n}$.

Proof. Since $S_{1}^{n}$ is not contractible in $S_{[1, \infty)}^{n} \cup A$ and $\left(S_{[1, \infty)}^{n} \cup A\right) \cap$ $D=C$, it follows that $S_{1}^{n}$ is not contractible in $X_{n}$.

Now suppose that $f: X_{n} \rightarrow X_{n}$ is a map whose fixed point set is precisely $S_{1}^{n}$. If $f(A) \subset A$, then $f$ has a fixed point in $A$. Since this is not possible, there is a point $p$ in $A$ such that $f(p) \in X_{n}-A$. Let $V$ be a contractible neighborhood of $f(p)$ in $X_{n}$, and let $\beta: V \times I \rightarrow V$ denote a homotopy which deforms $V$ to a point. Since $f$ is continuous at $p$, there is a neighborhood $U$ of $p$ in $X_{n}$ such that $f(U) \subset V$. Then, for some $r$ in $[1, \infty)$, we have $S_{r}^{n} \subset U$. Let $\alpha: S_{1}^{n} \times$ $I \rightarrow S_{[1, r]}^{n}$ be a homotopy which deforms $S_{1}^{n}$ onto $S_{r}^{n}$.

Define a homotopy $H: S_{1}^{n} \times I \rightarrow X_{n}$ by

$$
H(x, t)= \begin{cases}f(\alpha(x, 2 t)), & \text { (if } \left.0 \leqq t \leqq \frac{1}{2}\right), \\ \beta(f(\alpha(x, 1)), 2 t-1), & \text { (if } \left.\frac{1}{2} \leqq t \leqq 1\right)\end{cases}
$$

It is easy to check that $H$ is a homotopy which deforms $S_{1}^{n}$ to a point in $X_{n}$. This contradiction shows that $S_{1}^{n}$ is not a fixed point set of $X_{n}$.

4. Problem. Let us restrict our discussion to the class of compacta. Then all the examples of spaces having the complete invariance property which are mentioned in the introduction of this paper are ANR-spaces. In fact, the first three examples are ARspaces. In view of this, it seems appropriate to suggest the following problem.

Does every AR-space (ANR-space) have the complete invariance property?

\section{REFERENCES}

1. H. Robbins, Some complements to Brouwer's fixed point theorem, Israel J. Math., 5 (1967), 225-226. 
2. H. Schirmer, Properties of fixed point sets on dendrites, Pacific J. Math., 36 (1971), 795-810.

3. - Fixed point sets of homeomorphisms of compact surfaces, Israel J. Math., 10 (1971), 373-378.

4. — Fixed point sets of polyhedra, Pacific J. Math., 52 (1974), 221-226.

5. L. E. Ward, Jr., Fixed point sets, Pacific J. Math., 47 (1973), 553-565.

Received June 16, 1977. The research for this article was supported in part by the National Research Council of Canada (Grant A8205).

UNIVERSITY OF SASKaTCHEWAN

Saskatoon, Canada S7N 0W0 


\section{PACIFIC JOURNAL OF MATHEMATICS}

\section{EDITORS}

RICHARD ARENS (Managing Editor)

University of California

Los Angeles, California 90024

C. W. Curtis

University of Oregon

Eugene, OR 97403

C. C. MOORE

University of California

Berkeley, CA 94720

\section{J. DUGUNDJI}

Department of Mathematics University of Southern California Los Angeles, California 90007

R. Finn AND J. Milgram Stanford University Stanford, California 94305

ASSOCIATE EDITORS
E. F. BECKENBACH

B. H. NeUMANN

F. WOLF

K. YoshidA

\section{SUPPORTING INSTITUTIONS}

UNIVERSITY OF BRITISH COLUMBIA UNIVERSITY OF SOUTHERN CALIFORNIA CALIFORNIA INSTITUTE OF TECHNOLOGY STANFORD UNIVERSITY UNIVERSITY OF CALIFORNIA MONTANA STATE UNIVERSITY UNIVERSITY OF TOKYO UNIVERSITY OF NEVADA, RENO UNIVERSITY OF UTAH NEW MEXICO STATE UNIVERSITY OREGON STATE UNIVERSITY UNIVERSITY OF OREGON

WASHINGTON STATE UNIVERSITY UNIVERSITY OF WASHINGTON OSAKA UNIVERSITY 


\section{Pacific Journal of Mathematics}

\section{Vol. 74, No. $1 \quad$ May, 1978}

Gerald Arthur Anderson, Computation of the surgery obstruction groups

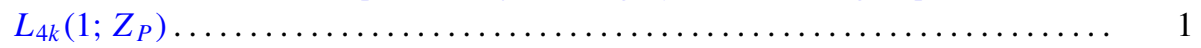

R. K. Beatson, The degree of monotone approximation ................ 5

Sterling K. Berberian, The character space of the algebra of regulated functions . . . 15

Douglas Michael Campbell and Jack Wayne Lamoreaux, Continua in the plane with

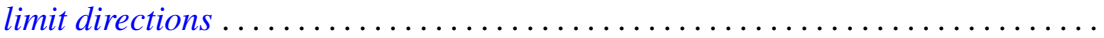

R. J. Duffin, Algorithms for localizing roots of a polynomial and the Pisot

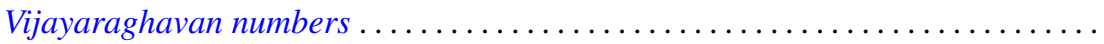

Alessandro Figà-Talamanca and Massimo A. Picardello, Functions that operate on

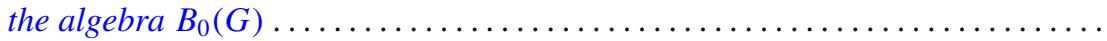

John Erik Fornaess, Biholomorphic mappings between weakly pseudoconvex

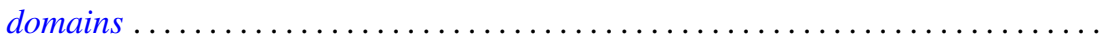

Andrzej Granas, Ronald Bernard Guenther and John Walter Lee, On a theorem of S.

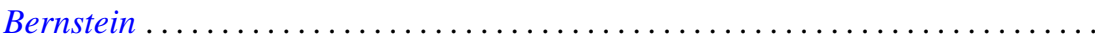

Jerry Grossman, On groups with specified lower central series quotients . .........

William H. Julian, Ray Mines, III and Fred Richman, Algebraic numbers, a constructive development . . . . . . . . . . . . . . . . . . . . . . .

Surjit Singh Khurana, A note on Radon-Nikodým theorem for finitely additive

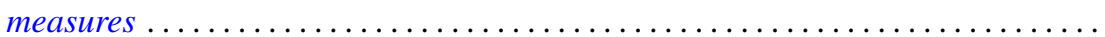

Garo K. Kiremidjian, A Nash-Moser-type implicit function theorem and nonlinear

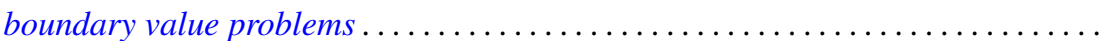

Ronald Jacob Leach, Coefficient estimates for certain multivalent functions ....

John Alan MacBain, Local and global bifurcation from normal eigenvalues. II . . 133

James A. MacDougall and Lowell G. Sweet, Three dimensional homogeneous algebras...

John Rowlay Martin, Fixed point sets of Peano continua ......

R. Daniel Mauldin, The boundedness of the Cantor-Bendixson order of some analytic sets...

Richard C. Metzler, Uniqueness of extensions of positive linear functions ..

Rodney V. Nillsen, Moment sequences obtained from restricted powers . .

Keiji Nishioka, Transcendental constants over the coefficient fields in differential elliptic function fields...

Gabriel Michael Miller Obi, An algebraic closed graph theorem

Richard Cranston Randell, Quotients of complete intersections by $\mathbf{C}^{*}$ actions . . 221

Bruce Reznick, Banach spaces which satisfy linear identities . .

Bennett Setzer, Elliptic curves over complex quadratic fields...

Arne Stray, A scheme for approximating bounded analytic functions on certain subsets of the unit disc.

Nicholas Th. Varopoulos, A remark on functions of bounded mean oscillation and bounded harmonic functions. Addendum to: "BMO functions and the

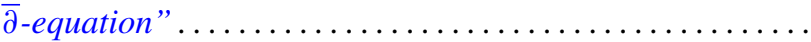

Charles Irvin Vinsonhaler, Torsion free abelian groups quasi-projective over their

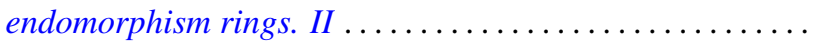

Thomas R. Wolf, Characters of $p^{\prime}$-degree in solvable groups ... 